\title{
Simulation in paramedic education - Is it the panacea?
}

\author{
Malcolm Boyle PhD \\ Affiliations: \\ Editor, Australasian Journal of Paramedicine
}

In the first main issue of 2014 we present the theme of simulation in paramedic education with three articles covering different aspects of simulation (1-3). There has been a push in recent years to increase the amount of simulation in paramedic courses, but at what cost and advantage? The study by Michau et al found that Monash University students had varying degrees of exposure to incident/condition types during their ambulance clinical placements, specifically, there was a lack of exposure to trauma-related cases (4). Therefore there is a need to bridge this gap in experience with simulation, but how?

As the article by Hagiwara et al highlights there are varying levels of fidelity used in simulation, but using these to enhance student skill levels, team work, situational awareness, and overall incident/condition management and the outcomes for patient care is yet to be fully evaluated. The study by Hagiwara et al reports on the use of simulation for Swedish ambulance nurses undertaking a prehospital trauma course and how the simulation component improves clinical competence of the ambulance nurses (2).

The study by Lazarsfield-Jensen and Bridges reports on a study that used simulation as a tool for interprofessional education with predominately paramedic students, nursing students, and general practitioner (GP) registrars. The study reported on the interaction between paramedics, nurses and GP registrars in a simulated GP practice room scenario, based in the Charles Sturt University Simulation Centre. The scenarios involved paediatric emergencies and investigated how the participants interacted and what they gained from the experience (3).

The study by Boyle et al used medium fidelity mannequins to assess a student's ability in measuring a variety of blood pressure (BP) readings. The study reported that students lacked accuracy in measuring low, normal and high BP readings and that the overall $\mathrm{BP}$ education process in the first year of the course needed to be reviewed (1).
The use of simulation centres for paramedic education at Charles Sturt University and Monash University (5) may assist in the realism of simulation but is there sufficient time in the curriculum to cater for the high number of students in the courses? Varying degrees of simulation may be a method for assessing student skill competence and their overall management of specific incidents/conditions, thereby ensuring the students are work ready. However, complex forms of simulation may work better with smaller student groups, especially when dealing with advanced clinical practice.

As we head into another year we invite you to partake in the unfolding story of simulation-based paramedic education.

\section{REFERENCES}

1. Boyle MJ, Williams B, Sawyer S. The accuracy of undergraduate paramedic students in measuring blood pressure: A pilot study. Australasian Journal of Paramedicine. 2014;11(2).

2. Hagiwara MA, Kängström A, Jonsson A, Lundberg L. Effect of simulation on the clinical competence of Swedish ambulance nurses. Australasian Journal of Paramedicine. 2014;11(2).

3. Lazarsfeld-Jensen A, Bridges D. Gaining a new respect for the other guys: Scripted scenarios for inter professional education in paediatric emergencies. Australasian Journal of Paramedicine. 2014;11(2).

4. Michau RA, Roberts S, Williams BA, Boyle MJ. An investigation of theory-practice gap in undergraduate paramedic education. BMC Med Educ. 2009;9(23).

5. Boyle MJ, Williams BA, Burgess SJ. Contemporary simulation education for undergraduate paramedic students. Emerg Med J. 2007;24(12):4. 